\title{
Correction to: Identity-based Multi-Recipient Public Key Encryption Scheme and Its Application in loT
}

\author{
Jing $\mathrm{Li}^{1,2} \cdot$ Xiangyan Tang ${ }^{3} \cdot{\text { Zhijun } \mathrm{Wei}^{4} \cdot \mathrm{Yu} \mathrm{Wang}^{1} \cdot \text { Wenbin Chen }}^{1,5}$ (D) $\cdot$ Yu-an $\operatorname{Tan}^{6}$ \\ Published online: 20 January 2020 \\ (C) Springer Science+Business Media, LLC, part of Springer Nature 2020
}

\section{Correction to: Mobile Networks and Applications https://doi.org/10.1007/s11036-019-01490-6}

The original version of this article unfortunately contained mistakes in the "Affiliation" and "Acknowledgements" sections. Author Jing Li's second affiliation "State Key Laboratory of Integrated Service Networks (ISN), Xidian University" and the sentence "This work was still supported by Open Research Project of State Key Laboratory of Integrated Service Networks (No. ISN20-10)" should be added in both sections, respectively.

The Affiliation and Acknowledgements sections are updated as below:

Acknowledgements This work was supported by National Natural Science Foundation of China (No. 61472091), Natural Science Foundation of Guangdong Province for Distinguished Young Scholars
(2014A030306020), Guangzhou scholars project for universities of Guangzhou (No. 1201561613) and Science and Technology Planning Project of Guangdong Province, China (2015B010129015), National Natural Science Foundation of China (No. 61702125, No. 61702126). This work was also supported by the Natural Science Foundation of China (U1936116), the Guangxi Key Laboratory of Cryptography and Information Security (GCIS201807), and by the Program for Innovative Research Team in Education Department of Guangdong Province Under No.2015KCXTD014. and No. 2016KCXTD017. This work was still supported by Open Research Project of State Key Laboratory of Integrated Service Networks (No. ISN20-10).

Publisher's note Springer Nature remains neutral with regard to jurisdictional claims in published maps and institutional affiliations.
The online version of the original article can be found at https:/doi.org/ 10.1007/s11036-019-01490-6

Wenbin Chen

cwb2011@gzhu.edu.cn

Jing Li

lijing@gzhu.edu.cn

Xiangyan Tang

997247@ hainu.edu.cn

Zhijun Wei

13823091082@139.cn

Yu Wang

yuwang@gzhu.edu.cn

Yu-an Tan

tan2008@bit.edu.cn
School of Computer Science, Guangzhou University, Guangzhou 510000, China

2 State Key Laboratory of Integrated Service Networks (ISN), Xidian University, Xi'an, China

3 School of Information Science and Technology, Hainan University, Hainan 570228, China

4 School of Astronautics, Beijing Institute of Technology, Zhuhai 519088, China

5 Guangxi Key Laboratory of Cryptography and Information Security, Guangxi 541004, China

6 School of Computer Science, Beijing Institute of Technology, Beijing 100000, China 